\title{
Spontaneous rupture of the spleen in rheumatoid arthritis
}

\author{
DORIAN O. HASKARD, CLARES. HIGGENS, L. N. TEMPLE, AND \\ H. L. F. CURREY
}

From the London Hospital Medical College, Turner Street, London E1 2AD

SUMMARY Two patients with rheumatoid arthritis without splenomegaly or Felty's syndrome developed spontaneous rupture of the spleen. The histological appearance was consistent with involvement of the splenic capsule by the rheumatoid process, and this may have predisposed to splenic rupture.

We report here 2 cases of rheumatoid arthritis in which spontaneous rupture of the spleen occurred. Neither patient had suffered any injury sufficient to explain the rupture, and in both the splenic parenchyma was normal. On the basis of the histological changes in the splenic capsules we believe that these ruptures were causally related to the rheumatoid disease. If so, this represents a manifestation of the disease which so far as we are aware has not previously been described.

\section{Case reports}

CASE 1

A 60-year-old maintenance engineer was admitted in April 1981 for investigation of a pleural effusion. For 10 years he had experienced episodic joint pains that were diagnosed as palindromic rheumatoid arthritis. He became seropositive in 1979 and was treated with nonsteroidal anti-inflammatory drugs. He did not receive steroids or long-term 'suppressive' therapy.

In the 3 months prior to admission he had noticed an increase in the joint pains, especially in the hands, wrists, and shoulders. His appetite was poor and he had lost $6 \mathrm{~kg}$ in weight. He complained of an unproductive cough, shortness of breath, and lethargy. There was no history of any contact with tuberculosis.

On examination he was emaciated. His temperature was $37 \cdot 5^{\circ} \mathrm{C}$. There were no palpable lymph nodes. In his chest there was evidence of bilateral pleural effusions. His liver was palpable $5 \mathrm{~cm}$ below the right costal margin. His spleen was impalpable. There was a rheumatoid subcutaneous nodule over the left elbow. The right shoulder was irritable, but there was no other evidence of active synovitis.
Investigations showed: $\mathrm{Hb} 13 \cdot 1 \mathrm{~g} / \mathrm{dl}$, white cell count $15.9 \times 10^{9} / 1$ ( $85 \%$ neutrophils), platelets 265 $\times 10^{9} / 1$, ESR $82 \mathrm{~mm} / \mathrm{h}$; a clotting screen was normal. Latex titre was greater than $1 / 320$. Antinuclear antibody was negative. Serum alkaline phosphatase was $328 \mathrm{IU} / 1$ (normal 30-85), gammaglutamyl transferase 75 IU/1 (normal less than 28), aspartate aminotransferase $20 \mathrm{IU} / \mathrm{l}$ (normal 5-40), bilirubin $7 \mu \mathrm{mol} / 1$ (normal less than 17).

Numerous sputum specimens were negative for acid fast bacilli and neoplastic cells. Mantoux test was negative at $1: 10000$ and positive at $1: 1000$. Chest $x$-ray showed a large right and a small left pleural effusion. In addition there was circular opacity $2 \mathrm{~cm}$ in diameter in the right mid zone, shown by tomography to be $17 \mathrm{~cm}$ from the posterior chest wall. $X$-rays of the hands showed an early erosion of the proximal interphalangeal joint of the left middle finger. A ${ }^{99 \mathrm{~m}} \mathrm{Tc}$ uptake scan showed the liver and spleen to be of normal size.

Two weeks after admission he complained of pain in the left hypochondrium. Palpation showed the spleen to be enlarged and tender. He developed a tachycardia and a falling blood pressure. Left iliac fossa tap revealed altered blood. At laparotomy he was found to have a splenic subcapsular haematoma with rupture into the peritoneum. Splenectomy was performed, and he made a good postoperative recovery. There had been no history of precipitating factors such as recent trauma, vomiting, or straining.

Pathological examination showed the spleen and subcapsular haematoma to weigh $300 \mathrm{~g}$. There was thickening of the splenic capsule by dense fibrous tissue. Granulation tissue was present both on the surface of the fibrous tissue and in relation to a solitary capsular rheumatoid nodule. The granulation tissue was composed of palisades of fibroblasts, lymphocytes, polymorph neutrophils, and foamy 
histiocytes, including occasional multinucleate forms (Figs. 1 and 2). The splenic pulp showed no significant abnormality.

Further investigation of the pleural effusion revealed a straw coloured aspirate with an undetectable glucose content and a protein concentration of $50 \mathrm{~g} / \mathrm{l}$. Cytology showed numerous degenerate polymorphs and macrophages, including mononuclear and multinucleated epithelium-like forms. There

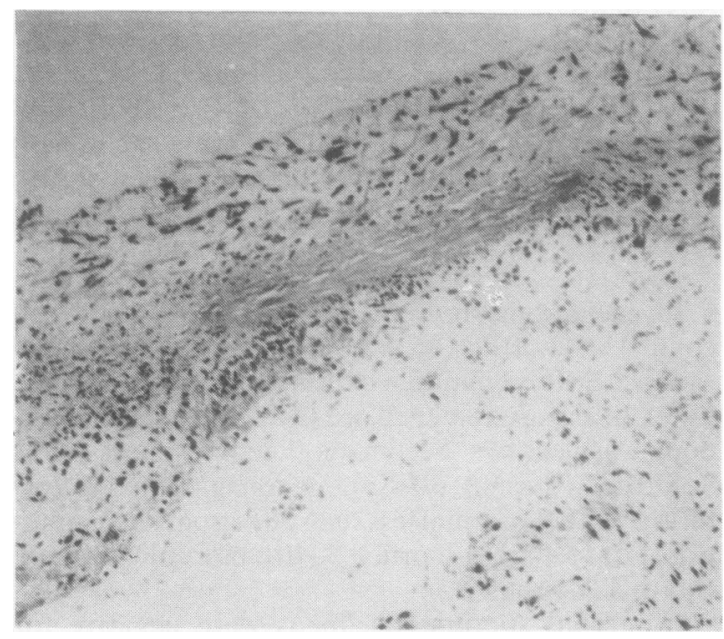

Fig. 1 Splenic capsule. (Haematoxylin and eosin; $\times 100$ original magnification.)

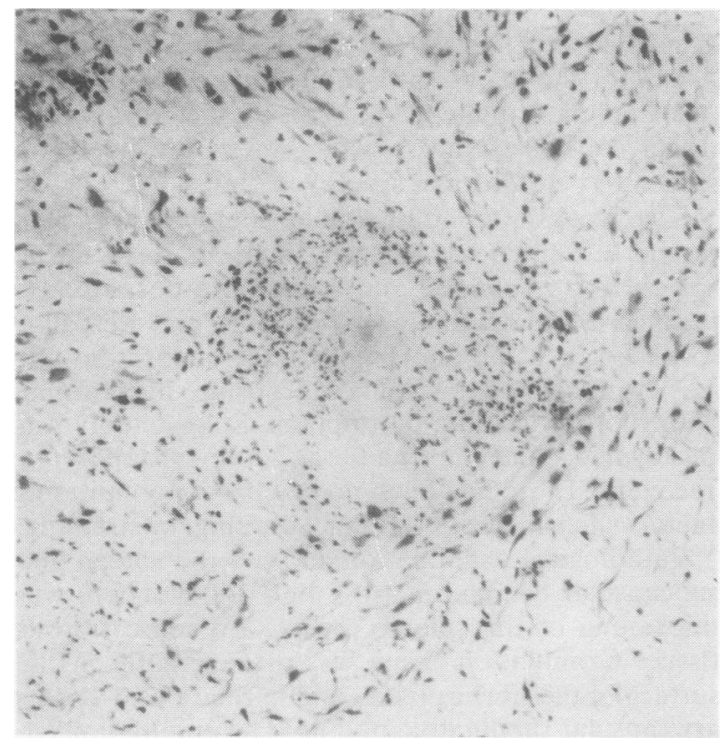

Fig. 2 Solitary capsular rheumatoid nodule.

(Haematoxylin and eosin; $\times 100$ original magnification.) were no organisms or malignant cells. This appearance is similar to that described in rheumatoid effusions. ${ }^{1}$

A pleural biopsy showed thickening by acellular hyaline fibrous tissue. A patchy lymphocytic infiltration was scattered throughout. Foamy histiocytes, including multinucleate forms, were present towards the pulmonary surface. Although nonspecific, the changes were consistent with that described in rheumatoid pleurisy ${ }^{2}$ and were comparable to the changes in the splenic capsule.

Bronchoscopy was normal and attempted percutaneous biopsy of the pulmonary opacity failed to provide a diagnostic sample.

The patient was treated empirically for 9 months with standard antituberculous chemotherapy, but there was no change in the coin lesion or the pleural effusions. These are believed to be a rheumatoid nodule and rheumatoid pleural effusion respectively.

\section{CASE 2}

A 67-year-old woman with a 7-month history of aggressive seropositive nodular and erosive rheumatoid arthritis was transferred to our care because of severe digital gangrene and multiple vasculitic ulcers of one month's duration. She had initially presented in April 1981 with proximal girdle pain, morning stiffness, and an ESR of $104 \mathrm{~mm} / \mathrm{h}$. A diagnosis was made of polymyalgia rheumatica, and she was started on prednisolone $160 \mathrm{mg}$ daily, reduced gradually after a month to $20 \mathrm{mg}$ per day.

At this lower dose the proximal muscle symptoms returned, with symmertical painful swelling of her hands. A clinical picture of rheumatoid arthritis then evolved, with widespread symmetrical polyarthritis involving metacarpophalangeal joints, proximal interphalangeal joints, wrists, shoulders, and ankles, together with multiple rheumatoid subcutaneous nodules. There was no evidence of vasculitis at that stage. Laboratory results included positive sheep cell agglutination test $(1: 5,120)$, negative antinuclear antibody test (ANA) and normal creatine kinase and thyroid function. An open synovial biopsy of her right acromioclavicular joint revealed pannus and granulation tissue typical of destructive rheumatoid arthritis.

In July 1981 she had increasing stiffness and weakness, with the appearance of further rheumatoid nodules. Penicillamine $500 \mathrm{mg}$ daily was started, and prednisolone continued.

In early October 1981 there was no symptomatic improvement. She had developed multiple pressure ulcers together with ulceration of rheumatoid nodules. Methylprednisolone given as pulsed intravenous infusions of $1 \mathrm{~g}$ daily for 3 days did not result in improvement. 
On 10 October 1981 she developed sudden pain and cyanosis in both hands. This progressed to distal digital gangrene of 3 fingers of her left hand. She later developed widespread vasculitic skin lesions on her finger pulps, nails, arms, and legs. She had experienced mild Raynaud's phenomenon for the past month, but there was no other history of previous peripheral vascular disease.

She was admitted on 30 October. Both hands were blue and cold with well demarcated distal gangrene of the 3 fingers of the left hand. She had widespread vasculitis, ulcerating nodules, and pressure ulcers. She also had marked proximal weakness with associated muscle pain. There was no clinical evidence of endocarditis or large blood vessel disease.

Investigation revealed ESR $130 \mathrm{~mm} / \mathrm{h}$, diffuse increase in serum immunoglobulins, cryoglobulins raised at $0.8 \mathrm{~g} / 1$ (normal less than $0.6 \mathrm{~g} / 1)$, creatine phosphokinase normal, latex test greater than 1:320, ANA negative.

Electromyographic studies showed evidence of myopathy of proximal muscles but without inflammatory features to suggest active polymyositis.

Immediate treatment included intravenous low molecular weight dextran for 48 hours, but the tips of the involved fingers remained gangrenous. Penicillamine was discontinued and she was started on azathioprine $2 \mathrm{mg}$ per body weight per day, with an increase in the dose of prednisolone to $40 \mathrm{mg}$ daily.

On 13 November her vasculitis and peripheral circulation had improved, but her muscle weakness persisted. Infection then became a major problem, and for this reason the azathioprine was discontinued.

She developed a Gram-negative septicaemia associated with a right lower lobe klebsiella pneumonia. Later, blood cultures also grew Staphylococcus aureus, similar to cultures from skin ulcer swabs. Pus also developed in her right wrist joint, and the previously well healed scar on her right acromioclavicular joint exuded pus, but this was repeatedly sterile. Treatment of her infections with appropriate intravenous antibiotics was moderately successful but also resulted in widespread overgrowth of candida, requiring intravenous miconazole.

On 18 November she developed respiratory distress with extensive dullness to percussion over the left lung. One litre of sterile straw-coloured fluid was aspirated. This produced temporary improvement, but she subsequently deteriorated and died on 9 December. Throughout the illness there was no trauma or violent vomiting, and she did not receive external cardiac massage.

At necropsy the macroscopic findings included a large left pulmonary embolus, a right lower lobe lung abscess, and a large perisplenic haematoma in rela- tion to 3 capsular tears in the spleen. The spleen weighed $150 \mathrm{~g}$ and was of normal consistency.

Histology showed thickening of the splenic capsule by dense, acellular fibrous tissue. Granulation tissue was present within and around the capsular tears, extending over the surface of the surrounding fibrous tissue. The granulation tissue was composed of palisades of fibroblasts, polymorph neurophils, foamy histiocytes, and multinucleate cells. The splenic parenchyma showed no abnormality.

There was no evidence of primary rheumatoid pleural or pericardial involvement, and skeletal muscles were microscopically normal.

\section{Discussion}

Spontaneous splenic rupture can occur as a complication of most diseases in which splenic involvement is present and has been reported as occurring rarely in the normal spleen. ${ }^{3}$ In our 2 cases there was no histological abnormality of the splenic parenchyma, and in particular no evidence of local sepsis, vasculitis, amyloid deposition, or neoplasia. There was, however, an abnormality of the splenic capsule resembling that previously described in a case of rheumatoid arthritis. ${ }^{4} \mathrm{~A}$ similar histological appearance can be found in the pleura and pericardium in cases of established rheumatoid pleurisy and pericarditis and may also be seen in very early rheumatoid synovitis. The appearance has been likened to that of an unfolded rheumatoid nodule. ${ }^{24}$ This appearance probably represents a non-organ-specific histological feature of the rheumatoid disease process which occurs in serosal surfaces as well as in nodules and synovial tissue.

In the case of rheumatoid pleurisy the surface layer of necrotic tissue is thought to give rise to the characteristic cytological picture of rheumatoid pleural fluid. ${ }^{1}$ It is of interest that these features were present in case 1.

Although we are not aware of splenic rupture having previously been described in association with rheumatoid arthritis, spontaneous hepatic rupture has been reported. ${ }^{5}$ The case described was thought to be due to an underlying arteritis.

On the evidence of our 2 cases it would appear that splenic capsular involvement by the rheumatoid process may predispose to rupture of the spleen in a manner comparable to the well recognised complication of rheumatoid lung and pleural nodules in which rupture leads to a sterile pyopneumothorax. ${ }^{6}$ Spontaneous rupture of the spleen should thus be added to the list of extra-articular manifestations of rheumatoid disease.

We thank Dr D. Golding for permission to report the second case. 


\section{References}

1 Boddington M M, Spriggs A I, Morton S A, Mowat A G. Cytodiagnosis of rheumatoid pleural effusions. J Clin Pathol 1971; 24: 95-106.

2 Champion G D, Robertson M R, Robinson R G. Rheumatoid pleurisy and pericarditis. Ann Rheum Dis 1968; 27: 521-9.
3 Cochrane J P S. Ruptured spleen. Br J Hosp Med 1980; 24: 398-404.

4 Gruenwald P. Visceral lesions in a case of rheumatoid arthritis. Arch Pathol 1948; 46: 59-67.

5 Hocking W G, Lasser K, Ungerer R. Spontaneous hepatic rupture in rheumatoid arthritis. Arch Intern Med 1981; 141: 792-4.

6 Davies D. Pyoneumothorax in rheumatoid lung disease. Thorax 1966; 21: 230-5. 